Review Article

\title{
Microbiota-gut brain axis involvement in neuropsychiatric disorders
}

\section{Background}

The microbiota-gut brain (MGB) axis is the bidirectional communication between the intestinal microbiota and the brain. An increasing body of preclinical and clinical evidence has revealed that the complex gut microbial ecosystem can affect neuropsychiatric health. However, there is still a need of further studies to elucidate the complex gene-environment interactions and the role of the MGB axis in neuropsychiatric diseases, with the aim of identifying biomarkers and new therapeutic targets, to allow early diagnosis and improving treatments.

\section{Areas covered}

To review the role of MGB axis in neuropsychiatric disorders, prediction and prevention of disease through exploitation, integration and combination of data from existing gut microbiome/microbiota projects and appropriate other International “-Omics” studies. We also evaluated the new technological advances to investigate the microbiome and evidence-based treatment modulating the gut microbiota through nutritional and other interventions.

\section{Expert Opinion}

The clinical studies have documented an association between alterations in gut microbiota composition and/or function, whereas the preclinical studies support a role for the gut microbiota in impacting behaviours which are of relevance to psychiatry and other central nervous system (CNS) disorders. Targeting MGB axis could be an additional approach for treating CNS disorders and all conditions in which alterations of the gut microbiota are involved.

Keywords: Ketogenic Diet; Microbiota-Gut Brain axis; Inflammation; Manipulating Microbiota; Metabolomics; Neuropsychiatric disorders. 


\section{Introduction}

Our consideration of the healthy human holobiont has greatly changed over the last years, due to the increased knowledge about the approximately $10^{14}$ cells of 1.000 different species, identified to date, in the gastrointestinal tract (GIT), collectively named the gut microbiota, and their potential functional roles[1]. The human gut microbiota consists mostly of bacteria, but also other microorganisms (i.e. archaea, fungi, parasites and viruses) resident in the GIT. Overall five phyla dominate the bacterial composition of the gut microbiota with the $90 \%$ of phylotypes identified in Firmicutes and Bacteroidetes, whereas Actinobacteria, Proteobacteria and Verrucomicrobia are minor components[2].

By including roughly 3.3 million non-redundant microbial genes, the gut microbiome (combined genetic material of the gut microbiota) has $~ 150$ times more genetic material than the human genome[3]. The gut microbiota is a highly dynamic system with its density and composition affected by many exogenous (e.g. diet, drugs, infections, environmental factors) and endogenous (e.g. age, sex, host genetic features) influencing factors[4]. Interesting, several classes of drugs impact on gut microbiota composition and not only antimicrobial agents; for example antipsychotics and antidepressant drugs can influence directly the gut microbiota[5]. All of the environmental factors may uniquely influence the microbiota composition, but it has been shown that the diet in particular plays a major role in determining the composition and changes of the gut microbiota[1].

It has been well studied that the gut microbiota contributes to regulate essential functions for host homeostasis, including metabolism, cardiovascular functions and immune/inflammatory processes[6]. Mapping the metagenome of hundreds of healthy individuals on the integrated catalogue of genes present in human gut microbiota revealed a large inter-individual variability in microbiota composition due to geographical and nutritional factors. Nevertheless, one third of the adult microbiota is similar within most individuals, therefore suggesting a compositional variability in which the microbiota stays within a functional range for the host. Divergence of 
microbiota from its functional range, initiating a condition known as dysbiosis, can be caused by one or more of these conditions: deep changes in diversity, loss of commensals, and bloom of pathobionts (members of the commensal microbiota that have the potential to induce pathology)[7]. An increasing body of evidence revealed that an altered gut-microbiota might be involved in several gastrointestinal/systemic diseases and in the individual's neuropsychiatric health, indeed the number of central nervous system (CNS) pathologies with associated compositional alterations in the gut microbiota is rapidly increasing (Table 1). The characterization of the host-microbiota communication pathways, above all the main microbiota-gut brain (MGB) axis network, remains a crucial step in the full understanding of human holobiont; establishing, interpreting and modulating symbiosis and dysbiosis will pave the way for future therapies[8]. This review is focused on the role of MGB axis in neuropsychiatric disorders, prediction and prevention of disease through exploitation, integration and combination of data from existing gut microbiome/microbiota projects and appropriate other International "-Omics" studies. We also addressed the impact of the recent technological advances to investigate the microbiome and evidence-based treatment modulating gut microbiota through nutritional and other interventions.

\section{The Microbiota-Gut Brain Axis}

Over the past decade, it has become clear that the MGB axis is a bidirectional communication pathway between gut bacteria and the CNS that exerts a profound influence on neural development, neuroinflammation, activation of stress response and neurotransmission, in addition to modulating complex behaviours, such as sociability and anxiety[9]. The gut microbiota can potentially influence, directly and indirectly, these central processes through immune system activation (e.g. inflammatory cytokines and chemokines)[10], production of neurotransmitters (e.g. serotonin, gamma-aminobutyric acid [GABA] and glutamate), short chain fatty acids (SCFAs) and key dietary amino acids as tryptophan (TRP) and its metabolites[10,11]. Furthermore, the gutmicrobiota can act through the permeability of gut barrier, with increase of circulating 
lipopolysaccharide (LPS)[13], modulating the levels of brain-derived neurotrophic factor (BDNF) and altering neuroendocrine (hypothalamic-pituitary-adrenal [HPA] axis)[14] and neural (e.g. vagus afferents, enteric nervous system) pathways[15] (Fig.1)(Table 2). On the other hand, the brain affects gut peristalsis, sensory and secretion function, mainly via the vagus nerve[16]. However, several and not yet fully understood mechanisms are involved in this complex bidirectional network in health and during diseases. Indeed, gut microbiota perturbations can lead to alterations of these pathways, that can precede the onset or leading to worsening of different neuropsychiatric disorders and their manifestations[15,17]. Recently, culture-independent sequencing techniques have added a new dimension to the study of gut microbiota and the challenge to analyse the large volume of sequencing data is increasingly addressed by the development of novel computational tools and methods.

\section{Technological Advances to Investigate the Gut Microbiota and the MGB Axis}

The vast majority of microbiota studies use one of two deep sequencing approaches: 16S ribosomal RNA amplicon sequencing or shotgun metagenomics sequencing. The 16S rRNA sequencing reveals the bacterial composition of a sample at the phylum to species level, while a metagenomics sequencing approach will reveal the entire genetic content of a sample. This latter approach allows the reconstruction of the bacterial composition of the sample up to the strain level and delivers information on the presence of metabolic pathways, i.e. the functional potential[18]. Several studies were performed to compare different next-generation sequencing strategies and platforms with vary results[19].

Until a few years ago, many parameters of the microbiota had been difficult to address, e.g. the spatial organisation of bacteria in the intestine, their real-time metabolic activity, or their fitness state and viability. Now, with the advent of sequencing-independent technologies, such parameters can be evaluated using gnotobiology in combination with fluorescent in situ hybridization (FISH)[20], novel bacterial genetic tools[21], metabolomics[22], and flow cytometry[23]. 
The gut microbiota exerts many of its effects on the host by producing metabolites that have recently been shown to penetrate the host even into remote organs such as the CNS[24]. While the metabolic activity of the microbiota is often inferred from the pathways present in metagenomics datasets, only recent advances in mass spectrometry-based metabolomics have provided definitive assessments of the metabolites present[25]. To definitely identify novel bacterial strains and to functionally characterise bacteria from the gut microbiota, their isolation and cultivation is essential. While it was previously assumed that the vast majority of intestinal bacteria is uncultivable, at present we can cultivate approximately more than $50 \%$ of the bacteria present in freshly collected human faeces[19]. Approximately $60 \%$ of the bacterial genera present in our intestine form spores which likely facilitates their host-to-host transmission[26]. Furthermore,three rapid PCR-based methods, the terminal-restriction fragment length polymorphism (T-RFLP)[27] and the temperature/denaturing gradient gel electrophoresis (DGGE/TGGE)[28,29] are used to generate a fingerprint of an unknown microbial community to provide a good basis for comparing communities, however these methods are now largely replaced by high throughput sequencing above mentioned[30]. Finally, flow cytometry has long been appreciated as a technology that delivers multi-parametric information on the single cell level at a high throughput and with the opportunity for physical separation of cells (FACS sorting). As a recent example, the diagnosis of intestinal inflammation in mice has been possible through cytometric finger printing of the microbiota based on DNA content and bacterial forward scatter (as a proxy for cell size)[23]. In conclusion, deep sequencing technologies have revolutionised the field of microbiota research but to fully understand the many aspects of host-microbial mutualism, complementary approaches are needed.

\subsection{Methods and Challenges of Standardization and Gnotobiology}

Nowadays, researchers are investing many resources to study the relationship between the microbiota and the host with the aim to better understand the pathogenesis of diseases in which the 
microbiota is involved[31]. However, it is hard for different research groups to look at the same biological conditions and to achieve the same observation even in the case of working with animal models. This is mainly due to the changes of the microbiota upon the interaction with different environmental factors, such as housing conditions, food, water, bedding, and temperature[32]. In most of the common research animal facilities, the experimental rodents are housed in individually ventilated cages (IVCs). This system uses HEPA-filters and individual air system shows some advantages in protecting the animals from all microorganisms coming from the external environment and from other neighbour cages, therefore limiting the spread of pathogenic infections[33]. In this sense, every single cage is a unique environment and changes in the microbiota happen randomly in any cage mainly due to handling procedure of the animal caretakers. Altogether, these factors greatly induce variability in the composition of the microbiota between animal facilities and research institutions, and even within the same animal facility $[34,35]$. To standardize the microbiota within the same animal facility, to reduce the variability between facilities and to allow correct interpretation of experimental results, the control for all the different non-genetic variables that can affect the gut microbiota of genetically identical mice is mandatory[36].

Simple methods, such as co-housing and littermate-controlled experiments, can be extremely helpful to standardize the experimental conditions[37,38]. In addition, also gnotobiology, i.e. the study of animals in a germ-free environment, may be a powerful system to standardise the experimental conditions. Gnotobiotic consortia are considered a pool of microorganisms with a defined composition that are vertically and horizontally transmissible, and stable over time under sterile conditions. To achieve this condition, it is mandatory to adopt certain standardised axenic handling and housing procedures (e.g. diet standardisation and housing in flexible film isolators)[39,40]; this has recently allowed to study the role of a single bacterium and a single bacterial antigen in maintaining host-immune homeostasis[41]. 


\subsection{Metabolomics Approach for Neurological Diseases}

Metabolomics is the study of the metabolome, defined as the set of metabolites (small molecules with molecular weight $<1.5 \mathrm{kDa}$ ) produced in a biological system[42]. The metabolome has a highly dynamic nature and represents a very rapid indicator of the modifications of a system. Therefore, metabolomics is considered the -Omics closest to phenotypic expression, because it reflects both the information contained in the genetic code and the influences derived from the interaction with the environment[22]. It has promising applications in the medical field such as allowing the identification of metabolic patterns of metabolites at single patient level, a useful tool to understand the aetiology of a disease and to follow its progression over time, especially in the context of multifactorial and chronic diseases. Furthermore, the identification of unknown metabolites may allow to formulate new pathogenic hypotheses.

Liquid or Gas Chromatography-Mass spectrometry (GS-MS) and Spectroscopy are the most frequently used methods. The large amount of data obtained is processed by appropriate software (Markerlynk) and then elaborated through multivariate statistical analysis methods. The final step is the chemical-physical identification of the metabolites. Structural hypotheses in this sense can be formulated by comparison with databases of metabolites such as Human Metabolome database (HMDB) and METLIN[43]. The most significant features obtained by metabolomics analyses in all evaluable studies about most important neuropsychiatric disorders are represented in Table 3.

These data are far to be definitive and univocal; this is due to a multitude of variables, primarily the complexity of the microbiota, the range of metabolites concentration and lack of standardization. Nevertheless, results previously published in the literature raised positive expectations[13]. In fact, by modifying the diet and/or the microbiota, it may be feasible to improve clinical outcome in the early phase of the disease and to improve the patients' quality of life. 


\section{MGB Axis in Neuropsychiatric Disorders}

Most of the neuropsychiatric disorders in children and adults are considered as multifactorial diseases prompted by environmental factors in genetically susceptible individuals[44]. However, additional work is needed to explain the exact complex gene-environment interactions and to characterize the potential gut microbiota alterations that can precede, or aggravate (likely contributing), the onset (establishment/progression) of the different neuropsychiatric disorders and their manifestations. Indeed, the very high degree of phenotypic heterogeneity in neuropsychiatric disorders originates from epigenetic modifications due to the interaction between environmental risk factors and susceptible genetic loci without involving alteration in nucleotide sequence[45,46]. Such epigenetic modifications may lead to DNA methylation and various other changes (e.g. phosphorylation and acetylation) that directly act on histone proteins with changes on genes transcription[47]. Evidence suggests that dysbiosis and altered gut permeability play a pathogenic role allowing the input of molecules (e.g. pro-inflammatory cytokines IL-6, TNF- $\alpha$ and IL-1 $\beta$ and several chemokines including MCP-1, CXCL-1 and MIP-1 $\alpha$ ) potentially neurotoxic in the blood stream and activating the immune system in an abnormal way[48]. Indeed, as mentioned, the gut microbiota has shown to have a direct influence on the brain by modulating the immune system; a ultra-low state of immune activation is always present in gut due to microbial cells that continually stimulate the immune system which, in turn, controls microbial populations. This relationship has the aim to reinforce immunity barrier and therefore the containment of the microorganism themselves[49,50].

Some clinical studies have shown that patients affected by neuropsychiatric disorders such as autism spectrum disorders (ASD), schizophrenia, major depressive disorder (recently reviewed by Chung et colleagues[51] and not detailed in our review), epilepsy, Alzheimer' and Parkinson' diseases or multiple sclerosis (MS), could have alterations in their gut microbiota composition and MGB axis, but if it is directly correlated or proportional to disorders' severity is not yet clarified[52,53]. Generally, they also experience an increased incidence of gastrointestinal 
functional discomfort such as constipation, diarrhoea, abdominal pain, heartburn or have irritable bowel syndrome as comorbidity[54,55]. Despite some studies in ASD[56,57] suggest that the improvements of GI symptoms matches to that of mental symptoms, it remains an open question. Further clinical and preclinical investigations are needed, to specifically identify and counteract a MGB axis dysregulation. Indeed, the microbiota, and as a consequence the MGB, is more accessible and modifiable than the human genome, providing potentially much needed new opportunities for preventing or treating neuropsychiatric disorders.

\subsection{MGB Axis in Autism Spectrum Disorders}

“Autism Spectrum Disorders" comprise a complex group of brain development disorders characterized by social and communication impairment along with presence of repetitive/restrictive behaviours and systemic comorbidities. Intestinal signs and symptoms, related with inflammation, are common features in children with ASD; almost half of them exhibit gastrointestinal dysfunction[58]. Despite is very challenging to determine the exact occurrence of GI disorders in these patients due to communication issues, diarrhoea, bloating and constipation are the most frequently symptoms reported and are frequently associated with behavioural and emotional symptoms[59]. The prevalence of gastro-intestinal symptoms suggest a possible link between the gut microbiota and ASD. Recent data indicated a significant dysbiosis and a change in the stability, $\alpha$ and $\beta$ diversity and composition of gut microbiota in ASD children compared to healthy controls age-matched[60]. Therefore, gastrointestinal dysfunction, mild intestinal inflammation and associated gut microbiota alterations could be considered as a complex pathophysiological process contributing to development and/or maintenance of disease. The balance of inflammatory cytokines is skewed in children with ASD who display gastro-intestinal symptomatology when compared to those children that did not[61] and the intestinal permeability seems to be increased[62]. The gut microbiota composition of ASD children has been found to differ from that of neuro-typical individuals[63]. Autistic symptoms have also been correlated to reduced diversity of gut 
microbiota[64] and there is growing evidence that improving behaviours in ASD might be achieved through modulation of the gut microbiota itself[65,66].

Dietary interventions have been already proposed as a therapeutic measure for ASD symptoms, indeed, at the time of diagnosis several children have been treated with different restrictive diets [67]. Despite clinical studies are still limited and not standardized, a treatment with probiotic supplementation could be a better and more manageable alternative. A mixture of Bifidobacteria, Streptococci and Lactobacilli is the most promising treatment for behavioural symptoms and gastro-intestinal dysfunction in this patients[54]. Indeed, some studied showed that probiotic therapy with the above mentioned strains may improve GI dysfunction[68], positively change gut microbiota composition with normalization of Bacteroides/Firmicutes ratio and Desulfovibrio spp. abundance[69] and decrease the ATEC (Autism Treatment Evaluation Checklist) score[65] in children with ASD. Future research needs to assess contemporarily gut microbiota and metabolomic profiles, along with gastrointestinal symptoms and inflammation, behavioural and ASD symptoms, to obtain more complete information about their reciprocal relationships and to find possible different endo-phenotypes with specific therapeutic targets.

\subsection{MGB Axis in Schizophrenia}

Schizophrenia is a heterogeneous neurodevelopmental disorder with an annual incidence of 0.20/1,000 per year[70]. As well as ASD, the etiology of the schizophrenia and the relationship with MGB axis are still unknowns. However, complex environmental and genomic factors are involved and emerging pre- and clinical studies seem to demonstrate an association between an altered gut microbiota and schizophrenia, considering also the high rates of gastrointestinal disorders reported in schizophrenic patients[71].

A recent pilot study reported an altered gut microbiota composition in patients with a first-episode psychosis (increased abundance of Lactobacillaceae, Halothiobacillaceae, Brucellaceae, and Micrococcineae, and decreased Veillonellaceae) compared to healthy age-matched individuals[70]. 
These modifications were correlated positively with the severity of psychotic symptoms and the risk of remission at one year, whereas there was no correlation with response to antipsychotics treatment[70]. Finally, one interventional study using a mixture of probiotic (Lactobacillus rhamnosus and Bifidobacterium animalis) was unsuccessful to demonstrate an influence on positive or negative symptoms[72].

\subsection{MGB Axis in Neurodegenerative Disorders}

Alzheimer's (AD) and Parkinson's (PD) diseases are severe chronic neurodegenerative pathologies with dramatic consequences and a growing global incidence. Despite more than 50 years of intense research in the field, the available therapies are still only oriented towards symptomatic control. It is relevant to note that many neurodegenerative disorders share similar molecular mechanisms, involving misfolded protein aggregates, neuroinflammation and oxidative stress, suggesting that an hitherto unidentified upstream, common pathogenetic event might be involved[73]. Several studies suggest a possible "microbiota-approach" to neurodegeneration[74]. For instance, in PD the olfactory epithelium and the gut are early affected, two tissues with high concentration of micro-organisms[75,76]. Some studies analyzed the gut microbiota in PD patients with the aim to link progressive stages of disease with variations in gut microbiota composition and point out the differences compared to healthy controls[77]. A reduction in the abundance of Prevotellaceae/Prevotella spp. and Bacteroidaceae/Bacteroides spp. in fecal samples of PD patients as compared to healthy controls and an increase in relative abundance of Lactobacillus and Enterobacteriacae have been reported[78,79]. Furthermore, the increase of these latter has been correlated with the severity of motor deficit PD-related[80].

In $\mathrm{AD}$, the classic amyloid cascade hypothesis suggests that $\mathrm{A} \beta$ deposition is the first and main driver of neurodegeneration with neuroinflammation and neuronal loss; recently, it has been proposed that $A \beta$ is not the primum movens but it is released in response to a danger signal due to the presence or the recognition of other factors[81]. In this context, the microbiome has gathered 
interest: an altered gut-microbiota can lead to an immune system activation[82], start a local and peripheral pro-inflammatory state, with a consequently more permissive intestinal barrier that allows pro-inflammatory and bacteria mediators to easily leave the gut reaching the brain and causing neuroinflammation [83]. Moreover, $\mathrm{A} \beta$ has been seen acting as an antimicrobial peptide both in vitro and in vivo as it is capable of entrapping bacteria, fungi or some of their components[84]

Alterations in the gut microbiota have been highlighted in murine models of AD; for example the APP/PS1 mice had an inversed Bacteroides/Firmicutes ratio compared to controls[85]. Furthermore, in the same model, Minter et al., showed that circulating inflammation mediators and amyloid formation are influenced by an alteration of gut microbiota (increasing of Lachnospiraceae) antibiotics-induced[86].

Analogous finding has been found in AD patients with a reduced microbial diversity, a decrease number of Actinobacteria and Firmicutes (i.e. Ruminococcaceae, Clostridaceae, Turicibacteraceae) and an increase in Bacteroides compared to healthy individuals[87].

Finally, in the faecal microbiota of AD patients, a study showed a decreased concentration of Eubacterium rectale spp. and an increase in Shigella/Escherichia spp., with a potential overall proinflammatory effect compared to controls[88]. If impact of gut microbiota on chronic neurodegenerative diseases will be confirmed, our perspective in the diagnostic and therapeutic approach to these disorders will be completely changed.

\subsection{MGB Axis in Epilepsy}

Nowadays, few pre- and clinical studies are present in the literature regarding the potential influence of gut microbiota in epilepsy, a heterogeneous group of neurological disorders affecting 65 million people worldwide and characterized to the predisposition of epileptic seizures. These studies investigate the gut microbiota in a comparative manner (comparative analysis between germ free $[\mathrm{GF}]$ and colonized mice or healthy-patient groups) or with direct manipulation of gut 
microbiota (probiotics, prebiotics, antibiotics or ketogenic diet $[\mathrm{KD}$, see below] evaluating outcomes in the CNS (e.g. seizures frequency, quality of life questionnaire scores, epilepsy-related symptoms). Only one case report linking epilepsy and gut microbiota has been published[89].

Peng and colleagues, showed that $\alpha$-diversity is increased and gut microbiota composition is enriched in rare flora (i.e. Clostridium XVIII, Dorea, Coprobacillus, Ruminococcus, Akkermansia, Neisseria, Coprococcus) in drug-resistant, but not in drug-sensitive, patients compared to healthy controls, assuming a role of gut microbiota and MGB connections in the treatment response[90].

Moreover, a pilot, open label and prospective study has reported that a mixture of probiotic (eight bacterial subspecies of Lactobacillus, Bacteroides and Streptococcus spp.) reduce seizures frequency and improve quality of life in patients with drug resistant epilepsy[91]; unfortunately, the authors not performed a gut microbiota analysis. Anyway, the mechanisms that could mediate the potential direct or indirect influence of gut microbiota on epilepsy processes are still unknowns.

\subsection{MGB Axis in Multiple Sclerosis}

Multiple sclerosis is an immune-mediated neurologic disorder characterized by demyelination and axonal damage, divided in relapsing-remitting MS (RRMS), progressive-relapsing MS (PRMS), primary progressive MS (PPMS) and secondary progressive MS (SPMS)[92]. Several studies have been performed to analyse the gut microbiota in MS patients compared to healthy controls with various findings. In RRMS patients, in different studies, it was observed a decreased abundance of Parabacteroides, Clostridium, Faecalibacterium or Prevotella and an increase in Firmicutes (i.e. Dorea and Blautia)[93-96]. Other studies also showed a higher abundance of Akkermasia, Bilophila, Mycoplana, Pseudomonas, (Chen et al., 2016), and lower abundance of Sutterella in MS patients[93,97]. Finally, administration of different probiotic (Bifidobacterium animalis and bifidum, Streptococcus thermophiles and Lactobacillus spp.) in both mouse and rat models of experimental autoimmune encephalomyelitis (EAE) might reduce the symptoms and improve cognitive scores[98]. Overall, these findings showed that the gut microbiota in RRMS patient is 
characterized by an abundance of pro-inflammatory bacteria and, on the other hand, a decrease in bacteria responsible for T-regulatory and $\mathrm{CD} 4^{+}$responses with potential influence on $\mathrm{MGB}$ axis.

\section{The Role of the Diet in MGB Axis Modulation}

As a shared substrate between the host and the gut microbiota, diet affects the host via both direct intestinal absorption and microbial metabolite production with downstream effects. One example is the fermentation of dietary complex carbohydrates by gut microbiota to produce SCFAs that have nutritional, neuroactive and anti-inflammatory effects among others[99]. The development of a rich and stable gut microbiota is crucial for the proper development of many host physiologic functions. In the setting of disease, the dysbiosis is characterized by decreased diversity and the predominance of a few pathogenic taxa which can adversely affect host health. Gut microbiota act on health primarily through the metabolites they produce, which is strongly dependent upon the substrates available through dietary intake[100]. In this light, nutrition showed to be essential to maintain mental health. A dysregulated diet, leading to obesity and metabolic syndrome, is known to have detrimental effects on cognition, leading to inflammation, insulin resistance and an impairment of the blood brain barrier (BBB)[101].

There is now growing evidence, particularly for depression and schizophrenia, for an association between diet and mental health, independent to demographic and age factors or reverse causality[102]. This new field of research, focused to develop a rigorous evidence base to support the role of diet and nutrition in mental health, is named "Nutritional Psychiatry". Since the first study published in 2009 [103] examining the connection with diet quality and mental disorder, this field has grown rapidly, with observational studies performed in many different countries $[104,105]$.

Nutritional deficiencies due to insufficient intake or absorption of nutrients needful to human health are a recognized risk factor for psychiatric disorders. Indeed, blood levels of folate and B12 are reduced in schizophrenia and are related with symptoms severity[106]. Restore vitamin-B 
levels with supplementations could reduce symptoms and reverse some neurological deficits disorder-associated[106]. These supplements in nutrition interventions have often used to elude the difficulties of dietary intakes; a recent meta-analysis of Sarris et al. confirmed the efficacy of vitamin D, S-adenosyl-methionine, omega-3 and methyl-folate as adjunctive therapies for depression, but only limited support for zinc, folic acid, vitamin C, and tryptophan[107].

Finally, diet is also one of the major factors influencing the gut microbiota composition and metabolism, contributing to host homeostasis and disease susceptibility[108]; therefore, it is extremely important to understand how diet can influence the gut microbiota, and while it is clear that what we eat is standing at the nexus between the host and the microbiota, this process is far from clarified. Nowadays, despite the exact mechanisms of action are not well known, nutrition is a complementary and alternative approach in several neuropsychiatric diseases. Evidence to date indicates that a Mediterranean style diet is advisable for patients with mental disorders[109,110] and the KD is widely use in drugs-resistant epilepsy, as well as conditions ranging from ASD to chronic pain, MS and cancer[111].

\subsection{Ketogenic Diet and the MGB Axis}

The ketogenic diet is a high-fat and low-carbohydrate diet inducing ketone bodies production, mimicking the biochemical changes of starvation. It has been used in the treatment of epilepsy since the 1920s[112], including the metabolic disorders glucose transporter protein 1 deficiency syndrome (GLUT1-DS) and pyruvate dehydrogenase deficiency syndrome (PDHD)[113]. Randomized controlled trials in children with drug resistant epilepsy have reported $50 \%$ seizures reduction in $38-72 \%$ of patients[114]. Even though the KD has been used in the treatment of epilepsy for almost a century, the mechanism of action remains unclear. In the KD, $80-90 \%$ of the energy is derived from fat, and hence, initiation of the KD represents a major shift in macronutrient composition for most patients, a shift which is likely to impact the gut microbiota substantially. The extensive study by Olson et al.[115], provides hard evidence for both the impact 
of the KD on the gut microbiota, and for its necessity to observe the anti-seizures effect of the dietary treatment in two different mouse models of epilepsy. Furthermore, the study even suggests and provides evidence for the mechanism of the effect, i.e. through an increase in the hippocampal GABA/glutamate ratio.

The studies conducted so far in humans investigating the impact of the KD on the gut microbiota are limited by small sample sizes and different methods of bacteria measurements. Swidsinsky et al.[116] demonstrated a biphasic effect of a 1:1 ketogenic diet on 10 patients with MS.

Xie et al.[117] showed that the gut microbiota profile of infants with epilepsy comprise large numbers of pathogens, such as Streptococcus, which differed from that of healthy controls. After one week on the $\mathrm{KD}$, the gut microbiota changed significantly, with fewer pathogens and more beneficial bacteria. Furthermore, Zhang et al.[118] showed lower $\alpha$-diversity after KD therapy and revealed significantly decreased abundance of Firmicutes and increased levels of Bacteroidetes in children with epilepsy after six months on the diet. Tagliabue et al.[119] performed gut microbiota analysis in six patients with GLUT1-DS before and after three months on a classic KD. Compared to baseline, faecal microbial profiles revealed a statistically significant increase in Desulfovibrio spp., a bacterial group supposed to be involved in the exacerbation of the inflammatory condition of the gut mucosa associated to the consumption of fats of animal origin.

Recently, Lindefeldt et al. showed, after three months of KD treatment a decrease of Actinobacteria (i.e. Bifidobacterium genus, Longum and Adolescentis spp.), Eubacterium rectale and genus Dialister associated with an increase in Proteobacteria and genus Escherichia[120]. Then, the KD is associated with major changes in the gut microbiota composition and metabolism but remains to be determined the precise mechanisms by which the gut microbiota exerts its effect, especially in humans. 


\section{The Role of Probiotics and Prebiotics in MGB Axis Modulation}

In the last years, an increasing interest in using pre- and probiotics to optimize the gut microbiota, supported by evidence related with anxiety and stress, led to several authors to test these compounds in neuropsychiatric disorders.

According to World Health Organization (WHO) definition, probiotics are "Live microorganisms which, when administered/ingested in adequate amounts, confer a health benefit to the host"[121]. Probiotics are more specific than prebiotics, because there is less or none control on which specific bacteria will metabolize and consequentially proliferate using the prebiotics administrated. A preand probiotic combined compound, named symbiotic, might be more effective[122].

The probiotics are main composed by two bacteria genera: the lactic acid-producing bacteria Lactobacillus and Bifidobacterium. Some animal studies have shown the beneficial effect of probiotics for the treatment of many diseases (e.g. AD, ASD, epilepsy) and to improve cognition outcomes, but only few clinical studies in humans on the effectiveness of probiotics in neuropsychiatric conditions have been realized[123].

For example, Lactobacillus rhamnosus, helventicus and fermentum, have demonstrated to restore memory impairment stress-induced in mice[124] and spatial memory impairment due to microbiota alterations by ampicillin administration[125].

In patients with $\mathrm{AD}$, a randomized and double blind clinical trial, reported positive effects of a Lactobacillus subspecies combination (i.e. acidophilus, casei, bifidum, and fermentum) on cognitive functions[126]. Another randomized clinical trial controlled to placebo, has highlighted that a probiotic supplementation with Lactobacillus rhamnosus could reduce the risk of neuropsychiatric disorder development in childhood in 75 infants with ASD[127].

Furthermore, the effects of oral administrations of Lactobacillus and Bifidobacterium spp. rodent models of EAE or in patients with epilepsy or schizophrenia have been previously discussed[72,98]. 
Instead of probiotics or in combination with them, even prebiotics can be used to modulate the gut microbiota and the MGB axis. A prebiotic is "A substrate that is selectively utilized by host microorganisms and confers a health benefit" as proposed by the International Scientific Association for Probiotics and Prebiotics (ISAPP)[128]. These compounds comprise soluble fermentable fibres, non-digestible oligosaccharides (NDOs) and human milk oligosaccharides (HMOs).

Although prebiotic therapies potentially could be beneficial due to their enhancement of Lactobacilli and Bifidobacteria, few studies have been published regarding the beneficial effects of prebiotics on MGB axis both in animals and in humans. A study performed in male mice has showed that fructo-oligosaccharides (FOS), galacto-oligosaccharides (GOS) or a combination for 3 weeks, could have antidepressant and anxiolytic effects and a decreased stress-induced corticosterone release[129].

In humans, administration of N-Acetylcysteine (NAC; $1200 \mathrm{mg} / \mathrm{day}$ ) for 8 weeks induced a decrease of repetitive behavior and irritability in forty ASD infants compared to placebo group[130]. Finally, Grimaldi et al. reported that administering GOS in ASD children improve autism features probably due to an increased abundance of Bifidobacterium spp. and Lactobacillus spp.[131].

All together these findings seem to reveal that pre- and probiotics could be useful treatment options for neuropsychiatric disorders, but several studies, possibly randomized clinical trials, are mandatory to understand the mechanisms involved and the real effectiveness, bearing in mind that correlation doesn't implies causation.

\section{The Role of Faecal Microbiota Transplantation in MGB Axis Modulation}

The faecal microbiota transplantation (FMT) is a treatment indicated for severe Clostridium difficile infections and inflammatory bowel diseases that modifies substantially the gut microbiota composition and potentially correct its alterations[132]. Very few studies have been performed to test FMT efficacy in neuropsychiatric disorders[133]. 
In a study with rodents, the gut microbiota of PD patients is transferred by FMT in healthy mice with consequentially development of neuroinflammation and motor deficits[134]. Furthermore, FMT executed for constipation in three patients with MS and in two children with ASD has resulted in a significative improvement of neurological and autistic symptoms, respectively[135].

Moreover in ASD, a study with 18 children reported a decrease in behavioural and gastrointestinal symptoms for 8 weeks after a treatment with vancomycin for two weeks followed by FMT[136].

Finally, a case report in a patient with drug resistant epilepsy and Crohn's disease treated with FMT for the latter condition, achieved a seizures free condition without the need of antiepileptic treatment[89]. However, albeit FMT could potentially be advantageous for neuropsychiatric disorders, its clinical use is still far away.

\section{Expert Opinion}

Since the gut microbiota was first proposed to influence human health over one century ago, our understanding of its role has immensely improved. For the past decade, our scientific community has made astounding technological advances in microbiome characterization. Through basic science, translational, and clinical research, we have gained insight in brain-gut communication and enhanced our understanding of the complex multi-directional relationships that exist between gut, host and environment.

Multi-omics approaches based on the analysis of different body fluids and tissues with various profiling platforms have to potential to provide deeper insights into MGB axis disorders, including response to treatment and the contribution of environmental factors. Therefore, biomarker discovery experiments based on profiling approaches facilitated by recent technical development are likely to make a great contribution to uncovering disease mechanisms in complex neuropsychiatric disorders. Serious consideration should be given to the concurrent analysis of global metabolic changes peripherally (e.g. in blood) and centrally (in cerebrospinal fluid, CSF) to establish how closely abnormalities measurable in the blood are correlated to changes in the brain. 
Collectively, these technologies offer great promise for the identification of clinically relevant biomarkers for neurological and psychiatric conditions. Interventions with diet, pro- and prebiotics and faecal transplantation in animal model shave shown that the microbiota could play a role in mental health by regulating inflammatory and endocrine secretions, synthetizing neuroactive compounds and interacting with the vagus nerve.

Recent data suggest that a dysbiosis might lead to the production of neurotoxins, systemic inflammation and/or to the onset of a dysmetabolic syndrome with a generalized effect even at the brain level[137]. Inheritance, mode of infant delivery, but also age-related changes in adults strongly affect microbiota composition and might lead to the selective proliferation of less frequent strains that could result in unpredictable consequences even in distant organs or triggering a systemic negative response such as immune-depression or chronic inflammation[138,139]. Inflammation seems to be a critical pathophysiological feature of CNS disorders and may thus be a potential target for microbiota-based interventions. Neuroinflammation influences the prognosis of high-incidence disabilities as example, it is considered as one of the most important cause of idiopathic epilepsy, and it is associated to severe prognosis and drugs-resistance[140,141]. Due to a state of dysbiosis, a severe chronic inflammation background started in GIT would become systemic[17].

Neuro-mediated stimuli, pro-inflammatory cytokines, SCFAs, amino acids, endocrine neurotransmitters, reactive oxygen species and many other pathogenetic metabolites produced in GIT increase Intestine Mucous Barrier and BBB permeability allowing an activated immune system to trigger a chronic neuroinflammation state. Since the MGB axis is a bidirectional communication pathway, a chronic state of neuroinflammation promotes, at the same time, immune system dysregulation and gut microbiota alterations (Fig. 2). It is well known that patients affected by chronic, systemic inflammatory disease such as immunological, rheumatic or inflammatory bowel diseases, these last ones also characterized by a compositional microbiota 
alterations, have increased risk of developing neurological comorbidities[142]. In this light, targeting the MGB axis could be an additional approach not only for CNS disorders but for all disorders in which a gut microbiota alteration is involved.

\section{Five-years view}

In the next five years our knowledge on the microbiota and the MGB axis will undoubtedly increase significantly. The massive commitment of the scientific community and the development of new -Omics, will allow clarification of the pathophysiological mechanisms underlying the interaction between gut microbiota and local/systemic diseases (e.g. intestinal inflammatory diseases, neuropsychiatric disorders, metabolic syndromes). This could lead to a targeted therapy for the microbiota, preventing alterations or acting to restore normal intestinal flora.

In the next five years, we will probably have more specific medical treatments beyond pre- and pro-biotics supplements. The potential development of FMT treatment and the possibility to isolate single strains to be used as specific therapy is already underway and may become shortly available for clinical practice[143]. Furthermore, the findings on the exact function of the KD in epilepsy through the gut microbiota, could be used in other neuropsychiatric disorders but also in cancer and metabolic syndromes. Besides contributing to clinical management and treatment, identifying biomarkers of dysbiosis or symbiosis could help to prevent or recognise quickly several diseases. Moreover, the microbiome/microbiota mapping projects will allow, among other, to clarify the global inter-individual differences and detect microbiological profiles to be used as a healthy control. Therefore, future studies will be able to improve our knowledge and open up for new therapeutic options trough manipulation of the gut microbiota by dietary changes, specific pre- and probiotic supplements, or FMT. 


\section{Key issues}

- The gut microbiota is a highly dynamic and complex system with approximately $10^{14}$ cells of 1.000 different species.

- The host-microbiota communication pathways, above all the main microbiota-gut brain (MGB) axis network, are fundamental mechanisms to preserve a healthy human holobiont.

- Deep changes in diversity, loss of commensals, and bloom of pathobionts can cause a dysbiosis and the leak of microbiota from its functional range.

- Sequencing technologies are revolutionizing the field of microbiota research to fully understand the various aspects of host-microbial mutualism.

- Promising applications in the medical field is allowing the identification of metabolic patterns of metabolites at single patient level, a useful tool to understand the aetiology of a disease and to follow its progression over time.

- Gut microbiota can affect neural circuits and behaviour related to stress response and his compositional alterations can lead to an immune system activation, with local and systemic pro-inflammatory state and more permissive intestinal barrier.

- Targeted therapies focused on microbiota will likely play a large role in the future. 
Acknowledgments: "The Gut-Brain-Microbiome connection in neuropsychiatric diseases of children and adults" workshop (Genova, April 27-28th, 2018) was supported by Kolfarma, Fb Health, Ecupharma and by University of Genova (travel grant to PS). We also thank Leandro and Jacopo Nencioni (J Medical Books Ed. S.r.l.) for the support. 


\section{References}

[1] Zmora N, Suez J, Elinav E. You are what you eat: diet, health and the gut microbiota. Nat. Rev. Gastroenterol. Hepatol. 2018;

[2] Thursby E, Juge N. Introduction to the human gut microbiota. Biochem. J. 2017;474:18231836.

[3] Qin J, Li R, Raes J, et al. A human gut microbial gene catalogue established by metagenomic sequencing. Nature. 2010;464:59-65.

** Define and describe the minimal gut metagenome and the minimal gut bacterial genome in all individuals and most bacteria

[4] Lynch S V, Pedersen O. The Human Intestinal Microbiome in Health and Disease. Phimister EG, editor. N. Engl. J. Med. 2016;375:2369-2379.

[5] Maier L, Pruteanu M, Kuhn M, et al. Extensive impact of non-antibiotic drugs on human gut bacteria. Nature. 2018;555:623-628.

[6] Le Chatelier E, Nielsen T, Qin J, et al. Richness of human gut microbiome correlates with metabolic markers. Nature. 2013;500:541-546.

[7] Fischbach MA. Microbiome: Focus on Causation and Mechanism. Cell. 2018;174:785-790.

[8] van de Guchte M, Blottière HM, Doré J. Humans as holobionts: implications for prevention and therapy. Microbiome. 2018;6:81.

[9] Sherwin E, Dinan TG, Cryan JF. Recent developments in understanding the role of the gut microbiota in brain health and disease. Ann. N. Y. Acad. Sci. 2018;1420:5-25.

[10] Blander JM, Longman RS, Iliev ID, et al. Regulation of inflammation by microbiota interactions with the host. Nat. Immunol. 2017;18:851-860.

[11] Cryan JF, Dinan TG. Mind-altering microorganisms: the impact of the gut microbiota on brain and behaviour. Nat. Rev. Neurosci. 2012;13:701-712.

\section{** Review of the role of gut microbiota on brain and behaviour}

[12] Stilling RM, van de Wouw M, Clarke G, et al. The neuropharmacology of butyrate: The bread and butter of the microbiota-gut-brain axis? Neurochem. Int. 2016;99:110-132.

[13] Lin L, Zhang J. Role of intestinal microbiota and metabolites on gut homeostasis and human diseases. BMC Immunol. 2017;18:2.

[14] Farzi A, Fröhlich EE, Holzer P. Gut Microbiota and the Neuroendocrine System. Neurotherapeutics. 2018;15:5-22.

[15] Dinan TG, Cryan JF. The Microbiome-Gut-Brain Axis in Health and Disease. Gastroenterol. Clin. North Am. 2017;46:77-89.

[16] Bonaz B, Bazin T, Pellissier S. The vagus nerve at the interface of the microbiota-gut-brain 
axis. Front. Neurosci. 2018;12:1-9.

[17] Anderson G, Seo M, Berk M, et al. Gut Permeability and Microbiota in Parkinson's Disease: Role of Depression, Tryptophan Catabolites, Oxidative and Nitrosative Stress and Melatonergic Pathways. Curr. Pharm. Des. 2016;22:6142-6151.

[18] van den Elsen LW, Poyntz HC, Weyrich LS, et al. Embracing the gut microbiota: the new frontier for inflammatory and infectious diseases. Clin. Transl. Immunol. 2017;6:e125.

\section{*The role of gut microbiota in inflammatory and infectious diseases}

[19] Lau JT, Whelan FJ, Herath I, et al. Capturing the diversity of the human gut microbiota through culture-enriched molecular profiling. Genome Med. 2016;8:72.

[20] Mark Welch JL, Hasegawa Y, McNulty NP, et al. Spatial organization of a model 15member human gut microbiota established in gnotobiotic mice. Proc. Natl. Acad. Sci. U. S. A. 2017;114:E9105-E9114.

[21] Whitaker WR, Shepherd ES, Sonnenburg JL. Tunable Expression Tools Enable Single-Cell Strain Distinction in the Gut Microbiome. Cell. 2017;169:538-546.e12.

[22] Hollywood K, Brison DR, Goodacre R. Metabolomics: Current technologies and future trends. Proteomics. 2006;6:4716-4723.

[23] Zimmermann J, Hübschmann T, Schattenberg F, et al. High-resolution microbiota flow cytometry reveals dynamic colitis-associated changes in fecal bacterial composition. Eur. J. Immunol. 2016;46:1300-1303.

[24] Uchimura Y, Fuhrer T, Li H, et al. Antibodies Set Boundaries Limiting Microbial Metabolite Penetration and the Resultant Mammalian Host Response. Immunity. 2018;49:545-559.e5.

[25] Uchimura Y, Wyss M, Brugiroux S, et al. Complete Genome Sequences of 12 Species of Stable Defined Moderately Diverse Mouse Microbiota 2: TABLE 1. Genome Announc. $2016 ; 4$.

[26] Browne HP, Forster SC, Anonye BO, et al. Culturing of 'unculturable' human microbiota reveals novel taxa and extensive sporulation. Nature. 2016;533:543-546.

[27] Sjöberg F, Nowrouzian F, Rangel I, et al. Comparison between terminal-restriction fragment length polymorphism (T-RFLP) and quantitative culture for analysis of infants' gut microbiota. J. Microbiol. Methods. 2013;94:37-46.

[28] Hoyles L, Clear JA, McCartney AL. Use of denaturing gradient gel electrophoresis to detect Actinobacteria associated with the human faecal microbiota. Anaerobe. 2013;22:90-96.

[29] Filleron A, Simon M, Hantova S, et al. tuf-PCR-temporal temperature gradient gel electrophoresis for molecular detection and identification of staphylococci: Application to breast milk and neonate gut microbiota. J. Microbiol. Methods. 2014;98:67-75. 
[30] Khan F, Oloketuyi SF. A future perspective on neurodegenerative diseases: nasopharyngeal and gut microbiota. J. Appl. Microbiol. 2017;122:306-320.

[31] Cryan JF, Dinan TG. Talking about a microbiome revolution. Nat. Microbiol. 2019;4:552553.

[32] Pellegrini C, Antonioli L, Colucci R, et al. Interplay among gut microbiota, intestinal mucosal barrier and enteric neuro-immune system: a common path to neurodegenerative diseases? Acta Neuropathol. 2018;136:345-361.

* Alterations of the enteric bacterial-neuro-immune network related with the main neurodegenerative diseases

[33] Hecht G, Bar-Nathan C, Milite G, et al. A simple cage-autonomous method for the maintenance of the barrier status of germ-free mice during experimentation. Lab. Anim. 2014;48:292-297.

[34] Lundberg R, Bahl MI, Licht TR, et al. Microbiota composition of simultaneously colonized mice housed under either a gnotobiotic isolator or individually ventilated cage regime. Sci. Rep. 2017;7:42245.

[35] Hufeldt MR, Nielsen DS, Vogensen FK, et al. Variation in the gut microbiota of laboratory mice is related to both genetic and environmental factors. Comp. Med. 2010;60:336-347.

[36] Wullaert A, Lamkanfi M, McCoy KD. Defining the Impact of Host Genotypes on Microbiota Composition Requires Meticulous Control of Experimental Variables. Immunity. 2018;48:605-607.

[37] Lemire P, Robertson SJ, Maughan H, et al. The NLR Protein NLRP6 Does Not Impact Gut Microbiota Composition. Cell Rep. 2017;21:3653-3661.

[38] Mamantopoulos M, Ronchi F, McCoy KD, et al. Inflammasomes make the case for littermate-controlled experimental design in studying host-microbiota interactions. Gut Microbes. 2018;9:1-8.

[39] Macpherson AJ, McCoy KD. Standardised animal models of host microbial mutualism. Mucosal Immunol. 2015;8:476-486.

[40] Smith K, McCoy KD, Macpherson AJ. Use of axenic animals in studying the adaptation of mammals to their commensal intestinal microbiota. Semin. Immunol. 2007;19:59-69.

[41] Mooser C, Gomez de Agüero M, Ganal-Vonarburg SC. Standardization in host-microbiota interaction studies: challenges, gnotobiology as a tool, and perspective. Curr. Opin. Microbiol. 2018;44:50-60.

[42] Carraro S, Giordano G, Reniero F, et al. Metabolomics: A New Frontier for Research in Pediatrics. J. Pediatr. 2009;154:638-644. 
[43] Dettmer K, Aronov PA, Hammock BD. Mass spectrometry-based metabolomics. Mass Spectrom. Rev. 2007;26:51-78.

[44] Chin-Chan M, Navarro-Yepes J, Quintanilla-Vega B. Environmental pollutants as risk factors for neurodegenerative disorders: Alzheimer and Parkinson diseases. Front. Cell. Neurosci. 2015;9:124.

[45] Landgrave-Gómez J, Mercado-Gómez O, Guevara-Guzmán R. Epigenetic mechanisms in neurological and neurodegenerative diseases. Front. Cell. Neurosci. 2015;9:58.

[46] Uher R. Gene-Environment Interactions in Severe Mental Illness. Front. Psychiatry. 2014;5:48.

[47] Tordjman S, Somogyi E, Coulon N, et al. Gene $\times$ Environment interactions in autism spectrum disorders: role of epigenetic mechanisms. Front. psychiatry. 2014;5:53.

[48] Morris G, Fernandes BS, Puri BK, et al. Leaky brain in neurological and psychiatric disorders: Drivers and consequences. Aust. New Zeal. J. Psychiatry. 2018;52:924-948.

[49] Belkaid Y, Hand TW. Role of the microbiota in immunity and inflammation. Cell. 2014;157:121-141.

[50] Simeonova D, Ivanovska M, Murdjeva M, et al. Recognizing the leaky gut as a transdiagnostic target for neuro-immune disorders using clinical chemistry and molecular immunology assays. Curr. Top. Med. Chem. 2018;18.

[51] Cheung SG, Goldenthal AR, Uhlemann A-C, et al. Systematic Review of Gut Microbiota and Major Depression. Front. Psychiatry. 2019;10:34.

\section{** Review on link between major depression and gut microbiota}

[52] Fung TC, Olson CA, Hsiao EY. Interactions between the microbiota, immune and nervous systems in health and disease. Nat. Neurosci. 2017;20:145-155.

[53] Cenit MC, Sanz Y, Codoñer-Franch P. Influence of gut microbiota on neuropsychiatric disorders. World J. Gastroenterol. 2017;23:5486-5498.

[54] Fattorusso A, Di Genova L, Dell'Isola G, et al. Autism Spectrum Disorders and the Gut Microbiota. Nutrients. 2019;11:521.

[55] Severance EG, Yolken RH, Eaton WW. Autoimmune diseases, gastrointestinal disorders and the microbiome in schizophrenia: more than a gut feeling. Schizophr. Res. 2016;176:23-35.

[56] Kang D-W, Adams JB, Gregory AC, et al. Microbiota Transfer Therapy alters gut ecosystem and improves gastrointestinal and autism symptoms: an open-label study. Microbiome. 2017;5:10.

[57] Sanctuary MR, Kain JN, Chen SY, et al. Pilot study of probiotic/colostrum supplementation on gut function in children with autism and gastrointestinal symptoms. van Wouwe JP, 
editor. PLoS One. 2019;14:e0210064.

[58] Holingue C, Newill C, Lee L-C, et al. Gastrointestinal symptoms in autism spectrum disorder: A review of the literature on ascertainment and prevalence. Autism Res. 2018;11:24-36.

[59] Prosperi M, Santocchi E, Balboni G, et al. Behavioral Phenotype of ASD Preschoolers with Gastrointestinal Symptoms or Food Selectivity. J. Autism Dev. Disord. 2017;47:3574-3588.

[60] Adams JB, Johansen LJ, Powell LD, et al. Gastrointestinal flora and gastrointestinal status in children with autism - comparisons to typical children and correlation with autism severity. BMC Gastroenterol. 2011;11:22.

[61] Rose DR, Yang H, Serena G, et al. Differential immune responses and microbiota profiles in children with autism spectrum disorders and co-morbid gastrointestinal symptoms. Brain. Behav. Immun. 2018;70:354-368.

[62] de Magistris L, Familiari V, Pascotto A, et al. Alterations of the Intestinal Barrier in Patients With Autism Spectrum Disorders and in Their First-degree Relatives. J. Pediatr. Gastroenterol. Nutr. 2010;51:418-424.

[63] Kelly JR, Minuto C, Cryan JF, et al. Cross Talk: The Microbiota and Neurodevelopmental Disorders. Front. Neurosci. 2017;11:490.

[64] Kang D-W, Park JG, Ilhan ZE, et al. Reduced Incidence of Prevotella and Other Fermenters in Intestinal Microflora of Autistic Children. Gilbert JA, editor. PLoS One. 2013;8:e68322.

[65] Shaaban SY, El Gendy YG, Mehanna NS, et al. The role of probiotics in children with autism spectrum disorder: A prospective, open-label study. Nutr. Neurosci. 2018;21:676681.

[66] Patusco R, Ziegler J. Role of Probiotics in Managing Gastrointestinal Dysfunction in Children with Autism Spectrum Disorder: An Update for Practitioners. Adv. Nutr. 2018;9:637-650.

\section{*Probiotics in autism spectrum disorders}

[67] Millward C, Ferriter M, Calver S, et al. Gluten- and casein-free diets for autistic spectrum disorder. Cochrane database Syst. Rev. 2008;CD003498.

[68] Sichel J, Roberts E, Sichel LS, et al. Improvements in Gastrointestinal Symptoms among Children with Autism Spectrum Disorder Receiving the Delpro Probiotic and Immunomodulator Formulation. J. Probiotics Heal. 2013;01:1-6.

[69] Tomova A, Husarova V, Lakatosova S, et al. Gastrointestinal microbiota in children with autism in Slovakia. Physiol. Behav. 2015;138:179-187.

[70] Messias EL, Chen C-Y, Eaton WW. Epidemiology of schizophrenia: review of findings and 
myths. Psychiatr. Clin. North Am. 2007;30:323-338.

[71] Severance EG, Prandovszky E, Castiglione J, et al. Gastroenterology Issues in Schizophrenia: Why the Gut Matters. Curr. Psychiatry Rep. 2015;17:27.

[72] Dickerson FB, Stallings C, Origoni A, et al. Effect of Probiotic Supplementation on Schizophrenia Symptoms and Association With Gastrointestinal Functioning. Prim. Care Companion CNS Disord. 2014;16.

[73] Szeto JYY, Lewis SJG. Current Treatment Options for Alzheimer's Disease and Parkinson's Disease Dementia. Curr. Neuropharmacol. 2016;14:326-338.

[74] Westfall S, Lomis N, Kahouli I, et al. Microbiome, probiotics and neurodegenerative diseases: deciphering the gut brain axis. Cell. Mol. Life Sci. 2017;74:3769-3787.

[75] Silva de Lima AL, Hahn T, de Vries NM, et al. Large-Scale Wearable Sensor Deployment in Parkinson's Patients: The Parkinson@Home Study Protocol. JMIR Res. Protoc. 2016;5:e172.

[76] Sampson TR, Debelius JW, Thron T, et al. Gut Microbiota Regulate Motor Deficits and Neuroinflammation in a Model of Parkinson's Disease. Cell. 2016;167:1469-1480.e12.

\section{* In mice evidence of gut microbiota regulation in Parkison disease' symptoms}

[77] Radisavljevic N, Cirstea M, Finlay BB. Bottoms up: the role of gut microbiota in brain health. Environ. Microbiol. 2018;1462-2920.14506.

[78] Scheperjans F, Aho V, Pereira PAB, et al. Gut microbiota are related to Parkinson's disease and clinical phenotype. Mov. Disord. 2015;30:350-358.

[79] Qian Y, Yang X, Xu S, et al. Alteration of the fecal microbiota in Chinese patients with Parkinson's disease. Brain. Behav. Immun. 2018;70:194-202.

[80] Bedarf JR, Hildebrand F, Coelho LP, et al. Functional implications of microbial and viral gut metagenome changes in early stage L-DOPA-naïve Parkinson's disease patients. Genome Med. 2017;9:39.

[81] Heppner FL, Ransohoff RM, Becher B. Immune attack: the role of inflammation in Alzheimer disease. Nat. Rev. Neurosci. 2015;16:358-372.

[82] El Aidy S, Dinan TG, Cryan JF. Gut Microbiota: The Conductor in the Orchestra of Immune-Neuroendocrine Communication. Clin. Ther. 2015;37:954-967.

[83] Logsdon AF, Erickson MA, Rhea EM, et al. Gut reactions: How the blood-brain barrier connects the microbiome and the brain. Exp. Biol. Med. 2018;243:159-165.

[84] Kumar DKV, Choi SH, Washicosky KJ, et al. Amyloid- $\beta$ peptide protects against microbial infection in mouse and worm models of Alzheimer's disease. Sci. Transl. Med. 2016;8:340ra72-340ra72.

[85] Harach T, Marungruang N, Duthilleul N, et al. Reduction of Abeta amyloid pathology in 
APPPS1 transgenic mice in the absence of gut microbiota. Sci. Rep. 2017;7:41802.

[86] Minter MR, Zhang C, Leone V, et al. Antibiotic-induced perturbations in gut microbial diversity influences neuro-inflammation and amyloidosis in a murine model of Alzheimer's disease. Sci. Rep. 2016;6:30028.

[87] Vogt NM, Kerby RL, Dill-McFarland KA, et al. Gut microbiome alterations in Alzheimer's disease. Sci. Rep. 2017;7:13537.

[88] Cattaneo A, Cattane N, Galluzzi S, et al. Association of brain amyloidosis with proinflammatory gut bacterial taxa and peripheral inflammation markers in cognitively impaired elderly. Neurobiol. Aging. 2017;49:60-68.

[89] He Z, Cui BT, Zhang T, et al. Fecal microbiota transplantation cured epilepsy in a case with Crohn's Disease: The first report. World J. Gastroenterol. 2017;23:3565-3568.

[90] Peng A, Qiu X, Lai W, et al. Altered composition of the gut microbiome in patients with drug-resistant epilepsy. Epilepsy Res. 2018;147:102-107.

[91] Gómez-Eguílaz M, Ramón-Trapero JL, Pérez-Martínez L, et al. The beneficial effect of probiotics as a supplementary treatment in drug-resistant epilepsy: a pilot study. Benef. Microbes. 2018;9:875-881.

[92] Axisa P-P, Hafler DA. Multiple sclerosis. Curr. Opin. Neurol. 2016;29:345-353.

[93] Miyake S, Kim S, Suda W, et al. Dysbiosis in the Gut Microbiota of Patients with Multiple Sclerosis, with a Striking Depletion of Species Belonging to Clostridia XIVa and IV Clusters. Wilson BA, editor. PLoS One. 2015;10:e0137429.

[94] Cekanaviciute E, Pröbstel A-K, Thomann A, et al. Multiple Sclerosis-Associated Changes in the Composition and Immune Functions of Spore-Forming Bacteria. mSystems. 2018;3.

[95] Chen J, Chia N, Kalari KR, et al. Multiple sclerosis patients have a distinct gut microbiota compared to healthy controls. Sci. Rep. 2016;6:28484.

\section{*Evidence of different gut microbiota in multiple sclerosis patients}

[96] Cantarel BL, Waubant E, Chehoud C, et al. Gut Microbiota in Multiple Sclerosis. J. Investig. Med. 2015;63:729-734.

[97] Jangi S, Gandhi R, Cox LM, et al. Alterations of the human gut microbiome in multiple sclerosis. Nat. Commun. 2016;7:12015.

[98] Kwon H-K, Kim G-C, Kim Y, et al. Amelioration of experimental autoimmune encephalomyelitis by probiotic mixture is mediated by a shift in $\mathrm{T}$ helper cell immune response. Clin. Immunol. 2013;146:217-227.

[99] Shen T-CD. Diet and Gut Microbiota in Health and Disease. Nestle Nutr. Inst. Workshop Ser. 2017. p. 117-126. 
[100] David LA, Maurice CF, Carmody RN, et al. Diet rapidly and reproducibly alters the human gut microbiome. Nature. 2014;505:559-563.

[101] Proctor C, Thiennimitr P, Chattipakorn N, et al. Diet, gut microbiota and cognition. Metab. Brain Dis. 2017;32:1-17.

[102] Mörkl S, Wagner-Skacel J, Lahousen T, et al. The Role of Nutrition and the Gut-Brain Axis in Psychiatry: A Review of the Literature. Neuropsychobiology. 2018;1-9.

[103] Sánchez-Villegas A, Delgado-Rodríguez M, Alonso A, et al. Association of the Mediterranean dietary pattern with the incidence of depression: the Seguimiento Universidad de Navarra/University of Navarra follow-up (SUN) cohort. Arch. Gen. Psychiatry. 2009;66:1090-1098.

[104] Jacka FN. Nutritional Psychiatry: Where to Next? EBioMedicine. 2017;17:24-29.

[105] Marx W, Moseley G, Berk M, et al. Nutritional psychiatry: the present state of the evidence. Proc. Nutr. Soc. 2017;76:427-436.

\section{* Recent review on Nutritional Psychiatry}

[106] Firth J, Stubbs B, Sarris J, et al. The effects of vitamin and mineral supplementation on symptoms of schizophrenia: a systematic review and meta-analysis. Psychol. Med. 2017;47:1515-1527.

[107] Sarris J, Murphy J, Mischoulon D, et al. Adjunctive Nutraceuticals for Depression: A Systematic Review and Meta-Analyses. Am. J. Psychiatry. 2016;173:575-587.

[108] Heintz C, Mair W. You Are What You Host: Microbiome Modulation of the Aging Process. Cell. 2014;156:408-411.

[109] Bersani FS, Biondi M, Coviello M, et al. Psychoeducational intervention focused on healthy living improves psychopathological severity and lifestyle quality in psychiatric patients: preliminary findings from a controlled study. J. Ment. Health. 2017;26:271-275.

[110] Jacka FN, O’Neil A, Opie R, et al. A randomised controlled trial of dietary improvement for adults with major depression (the 'SMILES' trial). BMC Med. 2017;15:23.

[111] Boison D. New insights into the mechanisms of the ketogenic diet. Curr. Opin. Neurol. 2017;30:187-192.

[112] Wheless JW. History of the ketogenic diet. Epilepsia. 2008;49:3-5.

[113] Verrotti A, Iapadre G, Pisano S, et al. Ketogenic diet and childhood neurological disorders other than epilepsy: an overview. Expert Rev. Neurother. 2017;17:461-473.

[114] Martin K, Jackson CF, Levy RG, et al. Ketogenic diet and other dietary treatments for epilepsy. Cochrane Database Syst. Rev. 2016;2:CD001903.

[115] Olson CA, Vuong HE, Yano JM, et al. The Gut Microbiota Mediates the Anti-Seizure 
Effects of the Ketogenic Diet. Cell. 2018;173:1728-1741.e13.

\section{** Evidence that gut microbiota is necessary for ketogenic diet efficacy in epilepsy}

[116] Swidsinski A, Dörffel Y, Loening-Baucke V, et al. Reduced Mass and Diversity of the Colonic Microbiome in Patients with Multiple Sclerosis and Their Improvement with Ketogenic Diet. Front. Microbiol. 2017;8:1141.

[117] Xie G, Zhou Q, Qiu C-Z, et al. Ketogenic diet poses a significant effect on imbalanced gut microbiota in infants with refractory epilepsy. World J. Gastroenterol. 2017;23:6164-6171.

[118] Zhang Y, Zhou S, Zhou Y, et al. Altered gut microbiome composition in children with refractory epilepsy after ketogenic diet. Epilepsy Res. 2018;145:163-168.

[119] Tagliabue A, Ferraris C, Uggeri F, et al. Short-term impact of a classical ketogenic diet on gut microbiota in GLUT1 Deficiency Syndrome: A 3-month prospective observational study. Clin. Nutr. ESPEN. 2017;17:33-37.

[120] Lindefeldt M, Eng A, Darban H, et al. The ketogenic diet influences taxonomic and functional composition of the gut microbiota in children with severe epilepsy. npj Biofilms Microbiomes. 2019;5:5.

[121] Morelli L, Capurso L. FAO/WHO Guidelines on Probiotics. J. Clin. Gastroenterol. 2012;46:S1-S2.

\section{** International guidelines on Probiotics}

[122] Ford AC, Quigley EMM, Lacy BE, et al. Efficacy of Prebiotics, Probiotics and Synbiotics in Irritable Bowel Syndrome and Chronic Idiopathic Constipation: Systematic Review and Meta-analysis. Am. J. Gastroenterol. 2014;109:1547-1561.

[123] Liu X, Cao S, Zhang X. Modulation of Gut Microbiota-Brain Axis by Probiotics, Prebiotics, and Diet. J. Agric. Food Chem. 2015;63:7885-7895.

[124] Gareau MG, Jury J, MacQueen G, et al. Probiotic treatment of rat pups normalises corticosterone release and ameliorates colonic dysfunction induced by maternal separation. Gut. 2007;56:1522-1528.

[125] Wang H, Lee I-S, Braun C, et al. Effect of Probiotics on Central Nervous System Functions in Animals and Humans: A Systematic Review. J. Neurogastroenterol. Motil. 2016;22:589605.

[126] Akbari E, Asemi Z, Daneshvar Kakhaki R, et al. Effect of Probiotic Supplementation on Cognitive Function and Metabolic Status in Alzheimer's Disease: A Randomized, DoubleBlind and Controlled Trial. Front. Aging Neurosci. 2016;8:256.

[127] Pärtty A, Kalliomäki M, Wacklin P, et al. A possible link between early probiotic intervention and the risk of neuropsychiatric disorders later in childhood: a randomized trial. 
Pediatr. Res. 2015;77:823-828.

[128] Hill C, Guarner F, Reid G, et al. The International Scientific Association for Probiotics and Prebiotics consensus statement on the scope and appropriate use of the term probiotic. Nat. Rev. Gastroenterol. Hepatol. 2014;11:506-514.

[129] Burokas A, Arboleya S, Moloney RD, et al. Targeting the Microbiota-Gut-Brain Axis: Prebiotics Have Anxiolytic and Antidepressant-like Effects and Reverse the Impact of Chronic Stress in Mice. Biol. Psychiatry. 2017;82:472-487.

[130] Ghanizadeh A, Moghimi-Sarani E. A randomized double blind placebo controlled clinical trial of N-Acetylcysteine added to risperidone for treating autistic disorders. BMC Psychiatry. 2013;13:196.

[131] Grimaldi R, Cela D, Swann JR, et al. In vitro fermentation of B-GOS: impact on faecal bacterial populations and metabolic activity in autistic and non-autistic children. Marchesi J, editor. FEMS Microbiol. Ecol. 2017;93:fiw233.

\section{* Role of prebiotics in autism spectrum disorder}

[132] Borody TJ, Campbell J. Fecal Microbiota Transplantation. Gastroenterol. Clin. North Am. 2012;41:781-803.

[133] Xu M-Q, Cao H-L, Wang W-Q, et al. Fecal microbiota transplantation broadening its application beyond intestinal disorders. World J. Gastroenterol. 2015;21:102.

\section{* Review on faecal microbiota transplantation beyond intestinal infections and disorders}

[134] Sampson TR, Debelius JW, Thron T, et al. Gut Microbiota Regulate Motor Deficits and Neuroinflammation in a Model of Parkinson's Disease. Cell. 2016;167:1469-1480.e12.

[135] Borody TJ, Brandt LJ, Paramsothy S. Therapeutic faecal microbiota transplantation: current status and future developments. Curr. Opin. Gastroenterol. 2014;30:97-105.

[136] Kang D-W, Adams JB, Gregory AC, et al. Microbiota Transfer Therapy alters gut ecosystem and improves gastrointestinal and autism symptoms: an open-label study. Microbiome. 2017;5:10.

\section{* Study on faecal transplantation and autism disorder}

[137] Saad MJA, Santos A, Prada PO. Linking Gut Microbiota and Inflammation to Obesity and Insulin Resistance. Physiology. 2016;31:283-293.

[138] Boulangé CL, Neves AL, Chilloux J, et al. Impact of the gut microbiota on inflammation, obesity, and metabolic disease. Genome Med. 2016;8:42.

[139] Rana A, Musto AE. The role of inflammation in the development of epilepsy. J. Neuroinflammation. 2018;15:144.

[140] French JA, Koepp M, Naegelin Y, et al. Clinical studies and anti-inflammatory mechanisms 
of treatments. Epilepsia. 2017;58:69-82.

[141] Aronica E, Bauer S, Bozzi Y, et al. Neuroinflammatory targets and treatments for epilepsy validated in experimental models. Epilepsia. 2017;58:27-38.

[142] Morís G. Inflammatory bowel disease: an increased risk factor for neurologic complications. World J. Gastroenterol. 2014;20:1228-1237.

[143] Vindigni SM, Surawicz CM. Fecal Microbiota Transplantation. Gastroenterol. Clin. North Am. 2017;46:171-185.

[144] Berni Canani R, Paparo L, Nocerino R, et al. Gut Microbiome as Target for Innovative Strategies Against Food Allergy. Front. Immunol. 2019;10:191.

[145] Huang YJ, Marsland BJ, Bunyavanich S, et al. The microbiome in allergic disease: Current understanding and future opportunities - 2017 PRACTALL document of the American Academy of Allergy, Asthma \&amp; Immunology and the European Academy of Allergy and Clinical Immunology. J. Allergy Clin. Immunol. 2017;139:1099-1110.

[146] Mancuso C, Santangelo R. Alzheimer's disease and gut microbiota modifications: The long way between preclinical studies and clinical evidence. Pharmacol. Res. 2018;129:329-336.

\section{*Gut microbiota in Alzheimer'disease}

[147] Liu F, Li J, Wu F, et al. Altered composition and function of intestinal microbiota in autism spectrum disorders: a systematic review. Transl. Psychiatry. 2019;9:43.

[148] Wen C, Zheng Z, Shao T, et al. Correction to: Quantitative metagenomics reveals unique gut microbiome biomarkers in ankylosing spondylitis. Genome Biol. 2017;18:214.

[149] Breban M, Tap J, Leboime A, et al. Faecal microbiota study reveals specific dysbiosis in spondyloarthritis. Ann. Rheum. Dis. 2017;76:1614-1622.

[150] Tang WHW, Hazen SL. The Gut Microbiome and Its Role in Cardiovascular Diseases. Circulation. 2017;135:1008-1010.

[151] Li J, Zhao F, Wang Y, et al. Gut microbiota dysbiosis contributes to the development of hypertension. Microbiome. 2017;5:14.

[152] D'Argenio V, Casaburi G, Precone V, et al. No Change in the Mucosal Gut Microbiome is Associated With Celiac Disease-Specific Microbiome Alteration in Adult Patients. Am. J. Gastroenterol. 2016;111:1659-1661.

[153] Song M, Garrett WS, Chan AT. Nutrients, Foods, and Colorectal Cancer Prevention. Gastroenterology. 2015;148:1244-1260.e16.

[154] Kang M, Martin A. Microbiome and colorectal cancer: Unraveling host-microbiota interactions in colitis-associated colorectal cancer development. Semin. Immunol. 2017;32:3-13. 
[155] Franzosa EA, Sirota-Madi A, Avila-Pacheco J, et al. Gut microbiome structure and metabolic activity in inflammatory bowel disease. Nat. Microbiol. 2019;4:293-305.

[156] Pei L, Ke Y, Zhao H, et al. Role of colonic microbiota in the pathogenesis of ulcerative colitis. BMC Gastroenterol. 2019;19:10.

[157] Peng J, Pang N, Wang Y, et al. Next-generation sequencing improves treatment efficacy and reduces hospitalization in children with drug-resistant epilepsy. CNS Neurosci. Ther. 2018;

\section{* Evidence of gut microbiota involving in epilepsy}

[158] Staffas A, Burgos da Silva M, van den Brink MRM. The intestinal microbiota in allogeneic hematopoietic cell transplant and graft-versus-host disease. Blood. 2017;129:927-933.

[159] Yoshioka K, Kakihana K, Doki N, et al. Gut microbiota and acute graft-versus-host disease. Pharmacol. Res. 2017;122:90-95.

[160] Tap J, Derrien M, Törnblom H, et al. Identification of an Intestinal Microbiota Signature Associated With Severity of Irritable Bowel Syndrome. Gastroenterology. 2017;152:111123.e8.

[161] Xu D, Chen VL, Steiner CA, et al. Efficacy of Fecal Microbiota Transplantation in Irritable Bowel Syndrome. Am. J. Gastroenterol. 2019;1.

[162] Nallu A, Sharma S, Ramezani A, et al. Gut microbiome in chronic kidney disease: challenges and opportunities. Transl. Res. 2017;179:24-37.

[163] Qin N, Yang F, Li A, et al. Alterations of the human gut microbiome in liver cirrhosis. Nature. 2014;513:59-64.

[164] Ma J, Zhou Q, Li H. Gut Microbiota and Nonalcoholic Fatty Liver Disease: Insights on Mechanisms and Therapy. Nutrients. 2017;9:1124.

[165] Cekanaviciute E, Yoo BB, Runia TF, et al. Gut bacteria from multiple sclerosis patients modulate human T cells and exacerbate symptoms in mouse models. Proc. Natl. Acad. Sci. U. S. A. 2017;114:10713-10718.

[166] Berer K, Gerdes LA, Cekanaviciute E, et al. Gut microbiota from multiple sclerosis patients enables spontaneous autoimmune encephalomyelitis in mice. Proc. Natl. Acad. Sci. U. S. A. 2017;114:10719-10724.

[167] Le Chatelier E, Nielsen T, Qin J, et al. Richness of human gut microbiome correlates with metabolic markers. Nature. 2013;500:541-546.

[168] Bouter KE, van Raalte DH, Groen AK, et al. Role of the Gut Microbiome in the Pathogenesis of Obesity and Obesity-Related Metabolic Dysfunction. Gastroenterology. 2017;152:1671-1678.

[169] Maruvada P, Leone V, Kaplan LM, et al. The Human Microbiome and Obesity: Moving 
beyond Associations. Cell Host Microbe. 2017;22:589-599.

[170] Campos-Acuña J, Elgueta D, Pacheco R. T-Cell-Driven Inflammation as a Mediator of the Gut-Brain Axis Involved in Parkinson’s Disease. Front. Immunol. 2019;10:239.

[171] Kostic AD, Gevers D, Siljander H, et al. The Dynamics of the Human Infant Gut Microbiome in Development and in Progression toward Type 1 Diabetes. Cell Host Microbe. 2015;17:260-273.

[172] Forslund K, Hildebrand F, Nielsen T, et al. Disentangling type 2 diabetes and metformin treatment signatures in the human gut microbiota. Nature. 2015;528:262-266.

[173] Komaroff AL. The Microbiome and Risk for Obesity and Diabetes. JAMA. 2017;317:355.

[174] F€ Ulling C, Dinan TG, Cryan JF. NeuroView Gut Microbe to Brain Signaling: What Happens in Vagus. 2019;

[175] Bravo JA, Forsythe P, Chew M V, et al. Ingestion of Lactobacillus strain regulates emotional behavior and central GABA receptor expression in a mouse via the vagus nerve. Proc. Natl. Acad. Sci. U. S. A. 2011;108:16050-16055.

** Evidence of the important role of Lactobacillus strain in the bidirectional communication of the gut-brain axis

[176] De Vadder F, Grasset E, Mannerås Holm L, et al. Gut microbiota regulates maturation of the adult enteric nervous system via enteric serotonin networks. Proc. Natl. Acad. Sci. U. S. A. 2018;115:6458-6463.

[177] Sharon G, Sampson TR, Geschwind DH, et al. The Central Nervous System and the Gut Microbiome. Cell. 2016;167:915-932.

[178] Montiel-Castro AJ, González-Cervantes RM, Bravo-Ruiseco G, et al. The microbiota-gutbrain axis: neurobehavioral correlates, health and sociality. Front. Integr. Neurosci. 2013;7:70.

[179] Bravo JA, Forsythe P, Chew M V., et al. Ingestion of Lactobacillus strain regulates emotional behavior and central GABA receptor expression in a mouse via the vagus nerve. Proc. Natl. Acad. Sci. 2011;108:16050-16055.

[180] Erny D, Hrabě de Angelis AL, Jaitin D, et al. Host microbiota constantly control maturation and function of microglia in the CNS. Nat. Neurosci. 2015;18:965-977.

\section{**Host gut microbiota and CNS development}

[181] Obrenovich M. Leaky Gut, Leaky Brain? Microorganisms. 2018;6:107.

[182] Quigley EMM. Microbiota-Brain-Gut Axis and Neurodegenerative Diseases. Curr. Neurol. Neurosci. Rep. 2017;17.

[183] Yarandi SS, Peterson DA, Treisman GJ, et al. Modulatory effects of gut microbiota on the 
central nervous system: How gut could play a role in neuropsychiatric health and diseases. J. Neurogastroenterol. Motil. 2016;22:201-212.

[184] Ma Q, Xing C, Long W, et al. Impact of microbiota on central nervous system and neurological diseases: the gut-brain axis. J. Neuroinflammation. 2019;16:53.

[185] Cryan JF, Dinan TG. Microbiota and neuroimmune signalling-Metchnikoff to microglia. Nat. Rev. Gastroenterol. Hepatol. 2015;12:494-496.

\section{** Review on the microbiota gut brain axis}

[186] Kamada N, Seo SU, Chen GY, et al. Role of the gut microbiota in immunity and inflammatory disease. Nat. Rev. Immunol. 2013;13:321-335. 
Table 1. Examples of diseases for which alterations in gut microbiota composition have been evidenced in human clinical studies, next-generation-sequencing and representative references.

Pathologies

\begin{tabular}{|c|c|}
\hline Allergy & $\begin{array}{l}\text { Berni Canani et al., Front. in Immunol., 2019[144] ; Huang et al., } \\
\text { J Allergy Clin. Immunol., 2017[145] }\end{array}$ \\
\hline Alzheimer disease & $\begin{array}{l}\text { Cattaneo et al.,, Neurobiol Aging, 2017[88]; Mancuso and } \\
\text { Santangelo, Pharmacol. Res., 2018[146] }\end{array}$ \\
\hline Autism spectrum disorders & $\begin{array}{l}\text { Liu et al.,Transl Psychiatry, 2019[147]; Fattorusso et al., Nutrients. } \\
\text { 2019[54] }\end{array}$ \\
\hline Bone diseases & $\begin{array}{l}\text { Wen et al., Genome Biol, 2017[148]; Breban et al., Ann Rheum } \\
\text { Dis, 2017[149] }\end{array}$ \\
\hline Cardiovascular diseases & Tang et al., Circulation, 2017[150]; Li, Microbiome, 2017[151] \\
\hline Celiac disease & D’Argenio, Am J Gastroenterol, 2016[152] \\
\hline Colon-rectal cancer & $\begin{array}{l}\text { Song et al., Gastroenterology, 2015[153]; Kang and Martin, Semin } \\
\text { Immunol. 2017[154] }\end{array}$ \\
\hline Inflammatory bowel diseases & $\begin{array}{l}\text { Franzosa et al., Nat. Microb.,2019[155]; Pei et al., BMC } \\
\text { Gastroenterol. 2019[156] }\end{array}$ \\
\hline Major depressive disorder & Cheung et al., Front Psychiatry. 2019[51] \\
\hline Epilepsy & $\begin{array}{l}\text { Peng et al., Epilepsy Research, 2018[157]; Lindefeldt et al., Npj } \\
\text { Biofilms and Microbiomes[120] }\end{array}$ \\
\hline Graft versus host disease & $\begin{array}{l}\text { Staffas et al., Blood, 2017[158]; Yoshioka et al., Pharmacol. Res, } \\
\text { 2017[159] }\end{array}$ \\
\hline Irritable bowel syndrome & $\begin{array}{l}\text { Tap et al., Gastroenterology 2017[160]; Xu et al., Am J } \\
\text { Gastroent., 2019[161] }\end{array}$ \\
\hline Kidney diseases & Nallu et al., Transl Res. 2017[162] \\
\hline Liver pathologies & Qin et al., Nature, 2014[163]; Ma et al., Nutrients 2017,[164] \\
\hline Multiple sclerosis & $\begin{array}{l}\text { Cekanaviciute et al., PNAS USA, 2017[165]; Berer et al., PNAS } \\
\text { USA, 2017[166] }\end{array}$ \\
\hline Obesity & $\begin{array}{l}\text { Le Chatelier, Nature, 2013[167]; Bouter et al., Gastroenterology } \\
\text { 2017[168]; Maruvada et al., Cell Host Microbe. 2017[169] }\end{array}$ \\
\hline Parkinson disease & $\begin{array}{l}\text { Bedarf et al., Genome Med, 2017[80];Campos-Acuña et al., Front. } \\
\text { Immun., 2019[170] }\end{array}$ \\
\hline Diabetes & $\begin{array}{l}\text { Kostic et al., Cell Host Microbes, 2015[171]; Forslund et al, } \\
\text { Nature, 2015[172]; Komaroff, JAMA, 2017[173] }\end{array}$ \\
\hline
\end{tabular}


Table 2. Potential pathways involved in microbiota-gut brain communication.

\begin{tabular}{|c|c|}
\hline Pathways & Findings \\
\hline $\begin{array}{c}\text { Autonomic Nervous } \\
\text { System }\end{array}$ & $\begin{array}{l}\text { The vagus nerve is a major modulatory constitutive communication pathway between } \\
\text { the bacteria exposed to the gut and the brain; for example, chronic treatment } \\
\text { with Lactobacillus rhamnosus reduced stress-induced corticosterone and anxiety- and } \\
\text { depression-related behaviour via vagus nerve[174,175]. }\end{array}$ \\
\hline Enteric Nervous System & $\begin{array}{l}\text { The reintroduction of bacterial strains in germ free (GF) mice results in a rapid } \\
\text { increase in the circulating level of } 5 \text {-HT and other neuroactive metabolites, mediated } \\
\text { together with TRP, melatonin, gamma-aminobutyric acid and acetylcholine, by the } \\
\text { enteric nervous system[176,177]. }\end{array}$ \\
\hline Neuroendocrine System & $\begin{array}{l}\text { GF mice show hyper-response to stress, reversible by probiotic administration } \\
\text { suggesting the involvement of the endocrine system throughout the HPA axis that } \\
\text { affects stress response[178,179]. }\end{array}$ \\
\hline $\begin{array}{c}\text { Neuro-immune } \\
\text { Activation }\end{array}$ & $\begin{array}{l}\text { Gut-microbiota related alterations as an elevation in the circulating level of pro- and } \\
\text { anti-inflammatory cytokines such as IL-1, IL-6, TNF- } \alpha \text { and inflammation-related } \\
\text { proteins including lipopolysaccharides (LPS) are known to directly affect brain } \\
\text { functions, mood, behaviour and to elicit neuroinflammation[11,129]. }\end{array}$ \\
\hline $\begin{array}{c}\text { Gut-Microbiota } \\
\text { Metabolites }\end{array}$ & $\begin{array}{l}\text { Amyloid, short fatty acids (SCFAs) such as butyrate, able to control BDNF, } \\
\text { neurotoxins such as beta-metylamino-L alanine (BMAA) are produced by microbiota } \\
\text { strains, and, translocating from the intestinal mucosa to systemic circulation, might } \\
\text { interfere with the immune regulation and brain functionality[7]. For example, in GF } \\
\text { mice, systemic injection of butyrate showed antidepressant effect[12]. }\end{array}$ \\
\hline
\end{tabular}

5-HT serotonin; BDNF, brain-derived neurotrophic factor; GF, germ-free; HPA, hypothalamic-pituitary-adrenal; IL, interleukin; TNF, tumour necrosis factor; TRP, tryptophan. 
Table 3. Main metabolomic findings in neuropsychiatric disorders.

\begin{tabular}{|c|c|c|c|}
\hline Disease & $\begin{array}{l}\text { Significant gut microbiota } \\
\text { differences between patients } \\
\text { and controls }\end{array}$ & Significant differences of pathways/metabolites & $\begin{array}{c}\text { Papers } \\
\text { evaluated }\end{array}$ \\
\hline
\end{tabular}

\begin{tabular}{|c|c|c|c|}
\hline ASD & $\begin{array}{c}\uparrow \uparrow \text { Clostridium and Lactobacillus, } \\
\uparrow / \downarrow \text { Bacteroidetes, Firmicutes }\end{array}$ & 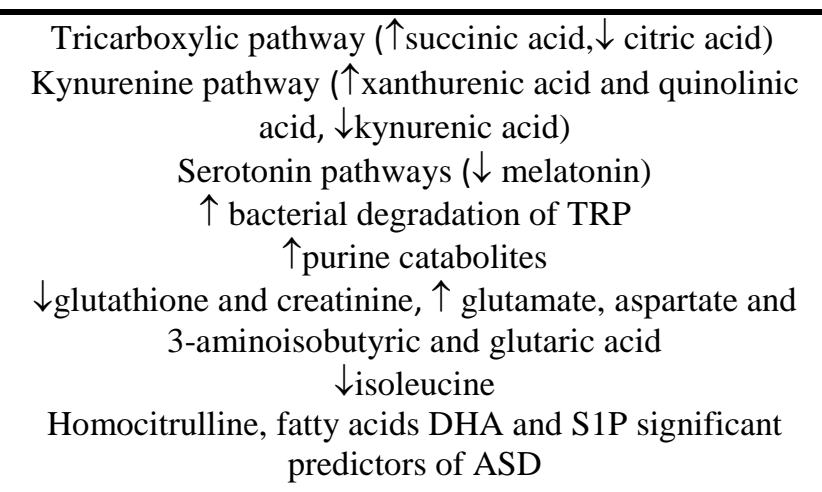 & 25 \\
\hline Schizophrenia & $\begin{array}{r}\uparrow \uparrow \text { Lactobacillaceae, } \uparrow \text { Firmicutes, } \\
\text { Halothiobacillaceae, Brucellaceae, } \\
\text { and } \\
\text { Micrococcineae, } \downarrow \text { Bacteroidetes, } \\
\text { Actinobacteria, Veillonellaceae }\end{array}$ & $\begin{array}{c}\text { Antioxidant pathway ( } \downarrow \text { uric acid and } \gamma \text { - } \\
\text { tocopherol, } \uparrow \text { allantoin) } \\
\uparrow \text { proline, glutamate and lactate } \\
\downarrow \text { arginine, glutamine, histidine andornithine } \\
\downarrow \text { phosphatidylcholine (C38:6) } \\
\uparrow \text { triglycerides } \\
\text { Glucose metabolism pathway ( } \uparrow \text { ribose 5-phosphate high } \\
\text { diagnostic performance for first-episode drug-naïve) }\end{array}$ & 14 \\
\hline ADHD & $\begin{array}{l}\text { No significant differences in } \\
\text { Firmicutes, Bacteroidetes, } \\
\text { Proteobacteria, and Actinobacteria } \\
\text { constituted the four dominant } \\
\text { phyla. } \\
\text { 个 Faecalibacterium }\end{array}$ & $\begin{array}{c}\uparrow \text { NGF } \\
\downarrow_{\text {cortisol }} \\
\downarrow \text { PUFA } \\
\uparrow \text { oxidative stress proteins (MAD, SOD, PON1, ARES, } \\
\text { TAS, TOS, OSI) } \\
\uparrow \text { adiponectin }\end{array}$ & 4 \\
\hline Epilepsy & $\begin{array}{l}\text { No significant differences in } \\
\text { Firmicutes and Bacteroidetes in } \\
\text { Drug-sensitive patients. } \\
\\
\text { In Drug-resistant patients } \\
\text { Firmicutes } \uparrow \text { and Bacteroides } \downarrow\end{array}$ & $\begin{array}{c}\downarrow \text { threonine, TRP and creatinine } \\
\text { lactate, glyceraldehyde and trans-13-octadecenoic acid in } \\
\text { serum jointly enabled a precision of } 92.9 \% \text { for } \\
\text { diagnosing seizures in generalized, secondary } \\
\text { generalized and partial seizures }\end{array}$ & 2 \\
\hline
\end{tabular}

PubMed database was used as the main systematic search engine to identify suitable studies on metabolomics in neuropsychiatric disorders up to October 2018. The following key search terms were used: "gut brain axis OR gut microbiota" AND "neuropsychiatric disorders autism OR autism spectrum disorders OR attention deficit hyperactivity disorder OR schizophrenia OR epilepsy" AND "metabolomics OR metabolome". References of included articles were also searched manually to identify relevant publications.

ASD, autism spectrum disorders; ADHD, attention deficit hyperactivity disorder; DHA, docosahexaenoic acid; NGF, nerve growth factor; PUFA, polyunsaturated fatty acid; TRP, tryptophan. 


\section{Legend of the figures}

Fig. 1. Schematic representation of microbiota gut brain axis [HPA, hypothalamic-pituitaryadrenal; SCFAs, short chain fatty acids][181-183]

Fig.2. MGB axis interactions: immune system dysregulation and gut microbiota composition alterations[184-186] 